\title{
Electrophysiological comparison study between effectiveness of the treatment modalities (Baclofen and Tizanidine drug) on spasticity management
}

\author{
By \\ Mohaimen A. Ridha ${ }^{* 1}$ \\ ${ }^{* 1}$ MBChB MSc, Neurophysiology Unit, Al-Imamain Al-Khadhimain Medical City, Baghdad Al- \\ Karkh Health Directorate, Ministry of Health and Environment, Baghdad, Iraq
}

\begin{abstract}
:
Background: Baclofen and tizanidine are both used for the treatment of muscle spasticity of spinal origin. patients and methods: This study was conducted in Ibn Al-Quf hospital for spinal cord injuries from the period December 2011 to June 2012. All of the participants gave written consent to participate in the study. The patients were divided into 2 groups: Group (I): Baclofen with physiotherapy treatment group; and Group (II): Tizanidine with physiotherapy treatment groups .H-reflex measurements were performed. parameters were studied: H-reflex latency, M wave latency, H-reflex conduction velocity, H-reflex duration, H-reflex amplitude. Results: All the patients had symptoms of spasticity at any time during the day with a Modified Ashworth Scale (MAS) before performing the H-reflex study. highly significant improvement in the H/M ratio when comparing positive controls to the two groups while the $\mathrm{H} / \mathrm{M}$ ratio in the negative controls shows no significant difference with group I and group II. A significant correlation was noticed between the height of control subjects \& H-reflex latency $(\mathrm{P}=0.002)$, significant positive correlation was also found $(\mathrm{P}=0.028)$ between the height \& $\mathrm{M}$ wave latency in the control subjects, The results revealed that the type of treatment did not affect the H-reflex and $\mathrm{F}$ wave parameters except for the H/M ratio. conclusion: H-reflex can provide information regarding neural function after spinal cord injury and the H/M ratio can be used as a good indicator for both spasticity assessment and response to treatment. Tizanidine hydrochloride is useful in the management of spasticity caused by SCI and can be used as a routine drug treatment although liver function tests should be periodically monitored.
\end{abstract}

keywords: Treatment modalities; Baclofen; Tizanidine; Spasticity management; H-reflex parameters 


\section{Introduction}

The muscle spindle is a specialized structure consisting of a connective tissue capsule surrounding several types of muscle fibers referred to as intrafusal muscle fibers ${ }^{(1)}$.

It is necessary to consider the morphology of the muscle spindle before describing their innervation, From the electrophysiological point of view, the $\mathrm{H}$ reflex has been considered the electrical equivalent of the tendon reflex. An important distinction between the H-reflex and the muscular contraction of a tendon tap is that the H-reflex directly activates the large Ia afferents in the nerve and bypasses the intrafusal muscle fibers, although some studies have shown patterns consistent with the reflex effects of both group Ia and group II afferents ${ }^{(2) .}$

The most commonly used H-reflex parameters are the H-reflex latency and the ratio of the peak-to-peak maximum $\mathrm{H}$-reflex amplitude to maximum $\mathrm{M}$ wave amplitude (H/M ratio). The H-reflex latency is directly related to the height and, to a lesser degree, age ${ }^{(3)}$. It is best predicted if these parameters are considered.

The aim of the study: The purpose of this study is to compare the effectiveness of the following treatment modalities on spasticity management using the H-reflex parameters in SCI persons: A) The central muscle relaxant (Baclofen) ; B) The central muscle relaxant (Tizanidine) .

\section{Subjects and methods:}

This study was conducted in Ibn Al-Quf hospital for spinal cord injuries from the period December 2011 to June 2012. All of the participants gave written consent to participate in the study.

They were having the following inclusion \& exclusion criteria:

(1) The presence of a traumatic lesion of the spinal cord before at least 3-6 months above the lumbar segments (above L2 spinal segment).

(2) Hyperreflexia in the triceps suraemuscle group (soleus \& gastrocnemius muscles) with spastic symptoms defined as being painful or restricting activities of daily life.

The patients were divided into 2 groups as group (I): Baclofen with physiotherapy treatment group; and group (II): Tizanidine with physiotherapy treatment group as well as control (Positive controls = patients without treatment, Negative controls = healthy). 
Clinical examination: All subjects were already examined neurologically for the spinal cord injury level by the rehabilitation specialists.

Spasticity was assessed according to the Modified Ashworth Scale, Knee and ankle joint reflexes were evaluated. Patients were inspected about the presence of pressure ulcers, muscle atrophy or contractures.

\section{H-reflex study}

H-reflex measurements were performed with 4-channels electrodiagnostic apparatus (CMS6600A EMG/EP system). Serial No. 20AB000006 Contec medical systems co., LTD. No.24 Huanghe West Road, Economic \& Technical Development Zone, Qinhuangdao, Hebei Province, 066004, P.R.China.

The following parameters were studied: H-reflex latency, $\mathrm{M}$ wave latency, $\mathrm{H}-$ reflex conduction velocity, H-reflex duration, H-reflex amplitude, $\mathrm{M}$ wave amplitude and the ratio between the maximum H-reflex amp. and the maximum $\mathrm{M}$ wave amp. was calculated (H/M ratio). These parameters were studied for the tibial nerve.

\section{Statistical analysis}

Data were analyzed using the Statistical Package for the Social Sciences (SPSS) version 16 for Windows (Norusis, 2008).

\section{Results:}

Number and percentage of patients in regard to their SCI level was as follows table 1.

Table (1): Number \& percentage of the spinal cord injury level in all patients' groups.

\begin{tabular}{|c|c|c|c|c|}
\hline & & \multirow{2}{*}{$\begin{array}{l}\text { Control } \\
\text { +ve controls } \\
\text { No. per. } \% \\
\end{array}$} & \multicolumn{2}{|c|}{ Study groups } \\
\hline & & & $\begin{array}{l}\text { Group I } \\
\text { No. per.\% }\end{array}$ & $\begin{array}{l}\text { Group II } \\
\text { No. per.\% }\end{array}$ \\
\hline $\begin{array}{l}\text { Spinal cord } \\
\text { injury level }\end{array}$ & $\begin{array}{l}\text { Cervical } \\
\text { Thoracic }\end{array}$ & $\begin{array}{ll}5 & 45.45 \% \\
6 & 54.54 \%\end{array}$ & $\begin{array}{ll}12 & 38.7 \% \\
19 & 61.3 \%\end{array}$ & $\begin{array}{cc}10 & 30.3 \% \\
23 & 69.7 \%\end{array}$ \\
\hline
\end{tabular}

Positive controls $=$ patients without treatment, Negative controls $=$ healthy, Group I = patients on physiotherapy and Baclofen drug, Group II = patients on physiotherapy and Tizanidine drug. 
All the patients had symptoms of spasticity at any time during the day with a Modified Ashworth Scale (MAS) before performing the H-reflex study as follows: (Table2).

Table (2): Number and percentage of the spinal cord injured patients according to the Modified Ashworth Scale

\begin{tabular}{|c|c|c|}
\hline MAS Grade & No. & Percentage \% \\
\hline Grade 0 & 9 & 15.25 \\
\hline Grade 1 & 33 & 55.93 \\
\hline Grade 2 & 8 & 13.55 \\
\hline Grade 3 & 4 & 6.78 \\
\hline Grade 4 & 2 & 3.4 \\
\hline Grade 5 & 3 & 5.09 \\
\hline
\end{tabular}

Results in table appearance highly significant improvement in the H/M ratio when comparing the positive controls to the two groups while the H/M ratio in the negative controls shows no significant difference with group I and group II. Mean $\pm \mathrm{D}$ of $\mathrm{H} / \mathrm{M}$ ratio for group I $(43.86 \pm 17.06)$ more than group II $(42.06 \pm 20.14)$ when compare with control.

Table (3): H/M ratio in the different treatment groups in comparison with the positive and negative controls.

\begin{tabular}{|l|l|l|}
\hline \multicolumn{2}{|c|}{ Groups } & $\begin{array}{l}\text { H/M ratio } \\
\text { Mean } \pm \text { D }\end{array}$ \\
\hline \multirow{2}{*}{ Control group } & +ve control & $72.94 \pm 12.93$ \\
& - ve control & $35.54 \pm 18.82 * *$ \\
\hline Group I & Group I & $43.86 \pm 17.06$ \\
& +ve controls & $72.94 \pm 12.93 * *$ \\
\cline { 2 - 3 } & Group I & $43.86 \pm 17.06$ \\
-ve controls & $35.54 \pm 18.82$ \\
\hline Group II & Group II & $42.06 \pm 20.14$ \\
\hline
\end{tabular}




\begin{tabular}{|l|l|l|}
\hline & +ve controls & $72.94 \pm 12.93^{* *}$ \\
\cline { 2 - 3 } & Group II & $42.06 \pm 20.14$ \\
-ve controls & $35.54 \pm 18.82$ \\
\hline
\end{tabular}

$* P<0.05, * *=P<0.001$

\section{Correlation of age and height with the H-reflex latency}

A significant correlation was noticed between the height of control subjects \& $\mathrm{H}$-reflex latency $(\mathrm{P}=0.002)$ (figure 1), while there was no significant correlation of age with the H-reflex latency $(\mathrm{P}=0.37)$ in this study.

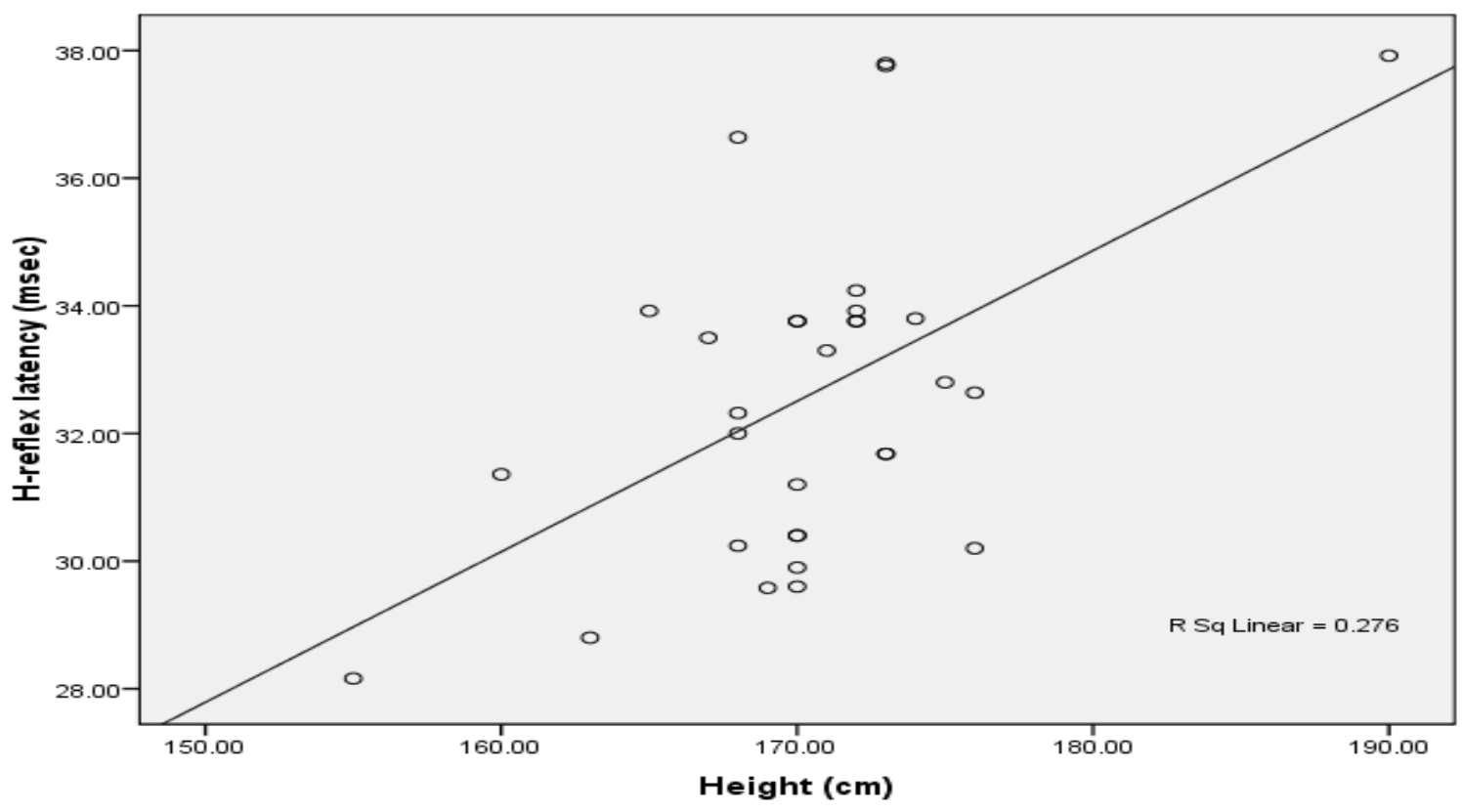

Figure (1): The significant positive correlation between height \& H-reflex latency in the control group.

Accordingly, a single linear regression equation was used from the obtained data to predict the optimal H-reflex latency for the patients \& controls to eliminate the effect of Ht on the H-reflex latency and as follows:

Optimal H-reflex latency $(\mathrm{msec})=-7.596+0.236(\mathrm{Ht}-\mathrm{cm})$

Similarly, a significant positive correlation was also found $(\mathrm{P}=0.028)$ between the height $\& \mathrm{M}$ wave latency in the control subjects as illustrated in figure (2).

Figure (2): Significant positive correlation between the height \& $M$ wave latency in the control group. 


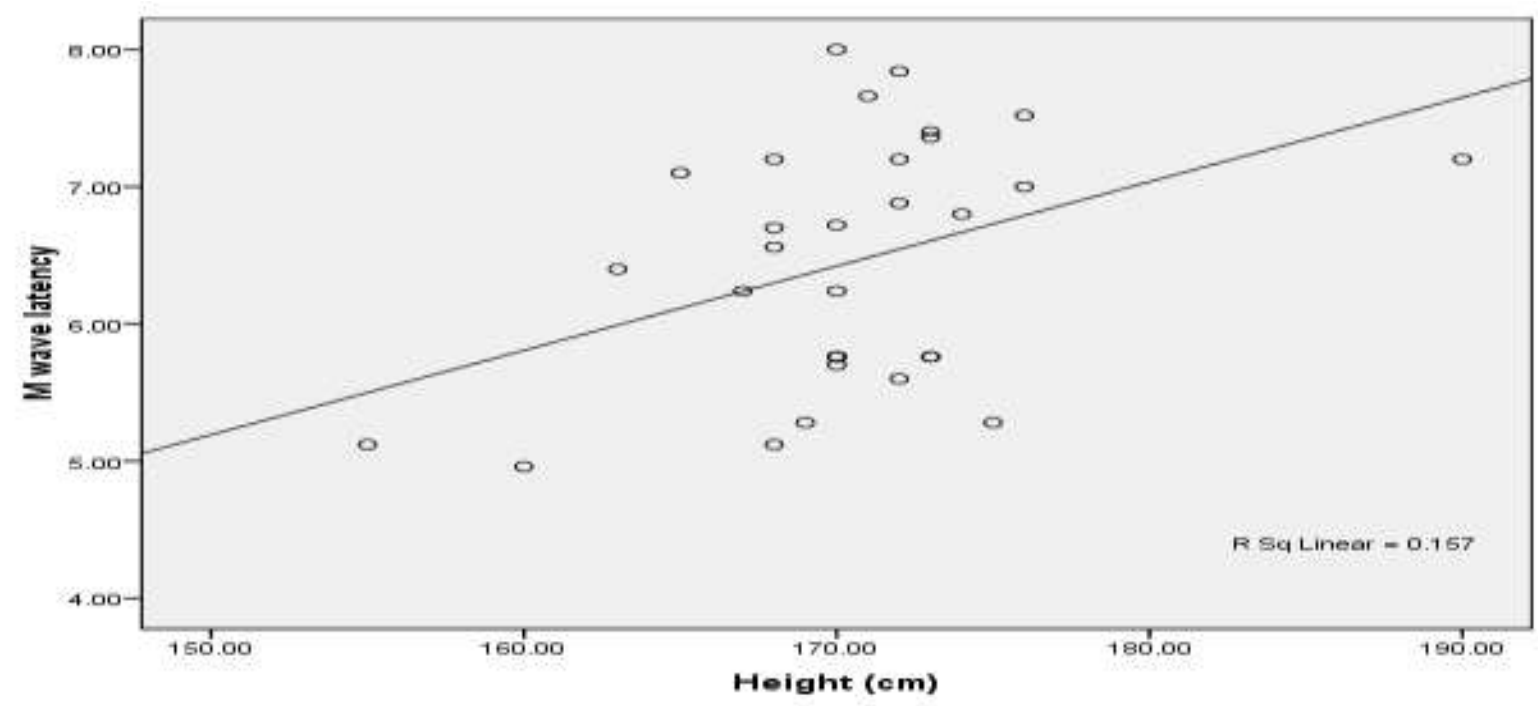

A single linear regression equation was also used from the obtained data to predict the optimal M wave latency for the patients \& controls and as follows:

Optimal M wave latency $(\mathrm{msec})=-4.028+0.061(\mathrm{Ht}-\mathrm{cm})$, The results revealed that the type of treatment did not affect the H-reflex and $\mathrm{F}$ wave parameters except for the $\mathrm{H} / \mathrm{M}$ ratio.

\section{Discussion:}

\section{Effect of extrinsic and intrinsic factors on H-reflex:}

The $\mathrm{H}$ reflex can be affected by various intrinsic and extrinsic factors. Extrinsic factors like temperature, electrical artifacts and poor recording techniques and intrinsic factors like age, sex, height, position of patient, sleep and Jendressik's maneuver $^{(\mathbf{4})}$ the extrinsic factors i.e., temperature, electrical artifacts and poor recording techniques were taken in consideration during the test, in such a way that we stabilized these factors.

On the other hand, in concern to the intrinsic factors, this study found a significant correlation between height and H-reflex latency, but there was no such a relation between age and weight with the H-reflex latency.

Correlation between height and H-reflex latency as found in this study was similar to many other studies ${ }^{(5,6,7)}$ Logically, taller subjects have longer conduction time of H-reflex because of longer conduction distance. 
On the contrary, there was no significant correlation between age and H-reflex latency and this was in contrary to other studies ${ }^{(4,7)}$ who found a significant correlation between age and H-reflex latency. They stated that in older ages, there will be an increase in H-reflex latency of $0.1 \mathrm{msec} / \mathrm{year}$. This may be due to multiple factors including drop-out of largest fibers, segmental demyelination and remyelination process with a consequent reduction in internodal length ${ }^{(8)}$.

This might be attributed to that the mean age of the control subjects was less than 40 years and this may minimize the effect of neural structure remodeling with increasing age. Similarly, weight had no significant correlation with H-reflex latency and this is also what had been obtained by ${ }^{(7)}$. Physiological variation in various parameters of $\mathrm{H}$ reflex due to sex could not be assessed due to very small sample size of female (control $=3,1 \%$ and patients $=5.9 \%$ ).

\section{H-reflex latency, duration, conduction velocity and F-wave latency.}

In this study the results of H-reflex latency, duration, conduction velocity and F-wave latency in spinal cord injured patients were not statistically different from the values obtained from healthy controls.

The SCI in this study was traumatic, there will be no reason that the peripheral nerve conduction velocity (NCV) will be affected in those patients.

Additionally, F-wave study reveals that the motor neurons are intact from the axon hillock of the anterior horn cell (AHC) down the motor axon to the innervated muscle ${ }^{(9)}$, the H-reflex latency was not affected by any treatment types used in this study. These findings were in agreement with many researchers ${ }^{(10,11)}$. They concluded that physiotherapy, drug treatment and TENS does not affect $\mathrm{H}$ reflex parameters significantly except for the $\mathrm{H} / \mathrm{M}$ ratio.

\section{H amp. max/ $M$ amp. max ratio (H/M ratio)}

The maximal $\mathrm{M}$ wave ( $\mathrm{M}$ max) amplitude indicates that all the $\alpha$-motoneurons of the innervated muscles were fired. It may be used as a base to normalize $\mathrm{H}$ reflex amplitude ${ }^{(12,13)}$. 
Change of H-reflex amplitude is dependent on the number of $\alpha$-motoneurons recruited by Ia afferents and affected by Ia inhibition ${ }^{(14) .}$ But the M wave max. amplitude is not changed because the maximal direct motor response represents the activity of the whole motoneuron pool. However, a recent study reported that changes of $\mathrm{M}$ wave max. amplitude in the soleus occurred with changes in the ankle joint angle and the level of muscular contraction ${ }^{(\mathbf{1 5})}$ and suggested that $\mathrm{M}$ wave max. amplitude is influenced by peripheral factors at the recording site such as muscle length and the level of voluntary muscular activation with a possibility that $\mathrm{M}$ wave max. amplitude changes with posture ${ }^{(\mathbf{1 6 )} \text {. }}$

Accordingly, the $\mathrm{H} \max / \mathrm{M}$ max ratio can be interpreted as the proportion of the entire motoneurone pool capable of being recruited.

\section{$\mathrm{H} / \mathrm{M}$ ratio of positive versus negative controls}

The results revealed that there was a highly significant difference between the positive and negative controls.

The high $\mathrm{H} / \mathrm{M}$ ratio observed in the positive controls was similar to many other studies ${ }^{(17 ;}$ 11). The H/M ratio in positive and negative control subjects was used as an index to measure the efficacy of the different treatment modalities used in this study as discussed below.

\section{$\mathrm{H} / \mathrm{M}$ ratio in group $\mathrm{I}$}

In this group, our results gained agreement with the findings of Seliem et al. (2007) and 10) that showed significant reduction in H/M ratio.

Moreover, Orsnes et al. (2000) suggested that the antispastic effect of Baclofen is not caused by an effect on the transmitter release from Ia afferents or on disynaptic reciprocal Inhibition ${ }^{(\mathbf{1 8})}$. But they suggest that the depression of the H-reflex by Baclofen is caused by a direct depression of motoneuronal excitability.

Additionally, Macdonell et al. suggested that the effects of Baclofen on spasticity and on the H-reflex may operate by different mechanisms and stated that not only the monosynaptic reflex contributes to spasticity ${ }^{(\mathbf{1 9})}$, but there are a number of other reflexes which also contribute. They suggested that oral Baclofen 
may inhibit polysynaptic reflexes in the spinal cord to reduce spasticity but may have little effect on monosynaptic reflex explaining the lack of inhibition of the $\mathrm{H}$ $\max / \mathrm{M}$ max. These findings are in contrary to our study. This can be explained by that in our study all the patients had taken their full therapeutic dose that resulted in depression of the monosynaptic reflex and consequently the $\mathrm{H} / \mathrm{M}$ ratio that was observed in another study after ITB bolus administration ${ }^{(20)}$.

\section{$\mathrm{H} / \mathrm{M}$ ratio in group II}

The findings of this group were in agreement with ${ }^{(21)}$ who stated that significant suppression of H-reflex activity was seen after Tizanidine treatment in animals with spinal ischemic injury at a dose of $1 \mathrm{mg} / \mathrm{Kg}$.

Moreover, previous experimental animal studies using systemically applied Tizanidine have demonstrated that Tizanidine preferentially depresses peripherally evoked spinal polysynaptic excitation and has modest suppressive effect on spinal monosynaptic reflexes ${ }^{(22) .}$ But Ismail et al. state that the centrally acting drug, Tizanidine, when administered at maximally tolerated dosage to patients had no significant effect on clonus ${ }^{(21)}$.

\section{Comparison of the different treatment groups}

There was no significant difference between the different treatment groups regarding the H/M ratio. However, in group I and group II, the H/M ratio reached nearly to the normal healthy subjects' value. This can be explained by the synergistic effect of $\mathrm{H}$ amplitude reduction and $\mathrm{M}$ amplitude increase (because of the combination treatment) resulting in overall decrease in $\mathrm{H} / \mathrm{M}$ ratio.

The results of the current study are in agreement with the findings of Wagstaff et al. who showed that improvement in muscle tone occurred in (60 to 82) $\%$ of tizanidine recipients compared with (60 to 65$) \%$ of baclofen ${ }^{(24)}$, that's mean Tizanidine has superior efficacy and less adverse effects than baclofen ${ }^{(25)}$.

Conclusion: H-reflex can provide information regarding neural function after spinal cord injury and the H/M ratio can be used as a good indicator for both spasticity assessment and response to treatment. Tizanidine hydrochloride is useful 
in the management of spasticity caused by SCI and can be used as a routine drug treatment although liver function tests should be periodically monitored.

\section{References:}

1) Barr ML, Kiernan JA. The Human Nervous System: An Anatomical Viewpoint, $5^{\text {th }}$ edition. Philadelphia, J.B. Lippincott Co. 1988: p. 40-43.

2) Al-Ani FS and Abd-Ali H. Reflex time in parkinsonian patients. J. Fac. Med. Baghdad. 2000; 42: 705-710.

3) Schimsheimer RJ, Ongerboer de Visser BW, Kemp B and Bour LJ. The flexor carpi radialis H-reflex in polyneuropathy: relations to conduction velocities of the median nerve and the soleus H-reflex latency. J Neurol Neurosurg Psychiatry. 1987; 50: 447-52.

4) Ghugare B , Singh R and Jain AP. Effect of physiological factors on soleus H-reflex in normal human subjects. J MGIMS. 2009; 14(i): 22 - 25.

5) Wu ZA and Chu FL. H reflex and F response- A study of 50 normal adults. Chin Med J (Taipei). 1982; 29: 271-7.

6) Lin KP, Chan MH and Wu ZA. Nerve conduction studies in healthy Chinese: correlation with age, sex, height and skin temperature. Chin Med J (Taipei). 1993; 52: 293-7.

7) Huang C, Chang W, Chang H, Tsai1 N and Lu C. Effects of Age, Gender, Height, and Weight on Late Responses and Nerve Conduction Study Parameters. Acta Neurologica Taiwanica. 2009; 18(4): 242-249.

8) Jacobs JM and Love S. Qualitative and quantitative morphology of human sural nerve at different ages. Brain. 1985; 108: 897-924.

9) Blum AS and Rutkove SB: The Clinical Neurophysiology Primer. Humana Press Inc., Totowa, NJ. 2007.

10) Seliem HA, Nagieb GS, Eliewa EA and Seliem AM. Efficacy of different modalities on spasticity management of spinal cord injury: clinical and electrophysiological study. Egypt Rheumatol Rehab. 2007; 34(3): 405416. 
11) Ayden G, serap T, Isk K, ozbudak DS, Sevim O. Transcutaneous electrical nerve stimulation versus Baclofen in spasticity: clinical and electrophysiologic comparison. Physical Medicine Rehabilitation. 2005; 84(8): 584-592.

12) Walton C, Kalmar J, Cafarelli E. Caffeine increases spinal excitability in humans. Muscle Nerve. 2003; 28: 359-364.

13) Nakazawa K, Miyoshi T, Sekiguchi H, Nozaki D, Akai M, Yano H. Effects of loading and unloading of lower limb joints on the soleus H-reflex in standing humans. Clin Neurophysiol. 2004; 115: 1296-1304.

14) Crone $\mathrm{C}$, Nielsen J. Spinal mechanisms in man contributing to reciprocal inhibition during voluntary dorsiflexion of the foot. $J$ Physiol. 1989; 416: 255-272.

15) Frigon A, Carroll TJ, Jones KE, Zehr EP, Collins DF. Ankle position and voluntary contraction alter maximal $\mathrm{M}$ waves in soleus and tibialis anterior. Muscle Nerve. 2007; 35: 756-766.

16) Takahara $\mathrm{T}$, Yamaguchi H, Seki K and Onodera S. Posture Induced Changes in the Maximal M-wave and the H-reflex Amplitude. Kawasaki Journal of Medical Welfare. 2011; 16(2): 50-56.

17) Maupas E, Marque P, Roques CF, Simonetta-Moreau M. Modulation of the transmission in group II heteronymous pathways by Tizanidine in spastic hemiplegic patients. J Neurol Neurosurg Psychiatry. 2004; 75: 130-135.

18) Orsnes G, Crone C, Krarup C, Petersen N and Nielsen J. The effect of Baclofen on the transmission in spinal pathways in spastic multiple sclerosis patients. Clin Neurophysiol. 2000; 111(8): 1372-9.

19) Macdonell RAL, Talalla A, Swash $M$ and Grundy D. Intrathecal Baclofen and the H-reflex. Journal of Neurology, Neurosurgery and Psychiatry. 1989; 52: 1110-1112.

20) Stokic DS, Yablon SA and Hayes A. Comparison of clinical and neurophysiologic responses to intrathecal Baclofen bolus administration in 
moderate to severe spasticity after acquired brain injury. Arch Phys Med Rehabil. 2005;86(9): 1801-6.

21) Fuchigami T, Kakinohana O, Hefferan MP, Lukacova N, Marsala S, Sugahara K, Yaksh TL and Marsala M. Potent suppression of stretch reflex activity after systemic or spinal delivery of Tizanidine in rats with spinal ischemia-induced chronic spastic paraplegia. Neuroscience. 2011; 194: 160 -169 .

22) Ono H, Fukushima $C$ and Fukuda H. Effect of the centrally acting muscle relaxant Tizanidine on spinal reflexes: involvement of descending noradrenergic systems. Jpn J Pharmacol. 1993; 62: 357-362.

23) Ismail B, Fugen O, Canan C, Mufit A and Hilmi U. Effect of Cold Application and Tizanidine on Clonus: Clinical and Electrophysiological Assessment. J Spinal Cord Med. 2009; 32(2): 132-139.

24) Wagstaff, A.J. and Bryson, H.M. (1997) Tizanidine a Review of Its Pharmacology, Clinical Efficacy and Tolerability in the Management of Associated with Cerebral and Spinal Disorders. Drugs, 53, 435-452.

25) Haque,N.; Saha N.; Ahmed T.; Debnath B.; Mollah A.H.; Rahman,E. Comparison of Baclofen and Tizanidine in Reducing Spasticity in Cerebral Palsy: A Randomized Control Trial. Open Journal of Pediatrics,2020. 10, 617-625). 